\title{
Mode Manipulation in Small Microsphere Systems
}

\author{
Yury P. Rakovich, Matthias Gerlach, John F. Donegan \\ Semiconductor Photonics Group, School of Physics and CRANN Research Centre, Trinity College Dublin \\ Dublin 2, Ireland \\ Tel: (+353) 896 1821, Fax: (+353) 35316711759, e-mail: Yury.Rakovich@tcd.ie
}

\begin{abstract}
In this work we demonstrate the possibility of mode manipulation in systems of symmetric photonic molecules formed by placing several microspheres in contact. We observe photonic nanojets that reflect the symmetry of the photonic molecule, with 3 jets located at 120 degrees with respect to each other for the triangular molecule. We also present some results showing the coexistence of whispering gallery modes and nanojets in the same structure.
\end{abstract}

Keywords: whispering gallery modes, spherical microcavities, photonic molecules, nanojets.

\section{INTRODUCTION}

Dielectric transparent microspheres are three-dimensional spherical microcavities which provide high Q-factors and a small mode volume leading to strong optical feedback within the cavity [1]. The optical resonances, also called whispering gallery modes (WGMs) are caused by total internal reflection of the light at the surface inside the sphere. As the electromagnetic field is not fully confined within the sphere, an evanescent field is present surrounding the spherical particle, which enables unique possibilities for interaction of the optical resonances with the surrounding medium or objects in close proximity to the cavity. Two or more microspheres close to each other allows optical coupling of the modes between the spheres which results in a complex rearrangement of the mode structure in the strong coupling regime similar to the electronic molecular orbitals in a chemical molecule. This leads to the notation of photonic molecules (PMs) [2] for coherently coupled microspheres. The optical modes in a coupled system of microcavities experience splitting into bonding and anti-bonding modes [3]. The so called supermodes in a photonic molecule are WGMs which extend over the whole structure of the coupled microcavities. Photonic molecule structures consisting of three or more microcavities were studied theoretically by different groups with regards to supermodes with dramatically increased Q-factors, low laser threshold and directional emission [4-6]. For optimal results, the structure requires a symmetric photonic molecule with size-matched microcavities and optimised gap alignment between the spheres [7]. In our recent studies of photonic molecules made of microspheres, we investigated optical coupling of a bi-sphere system [3]. In this present work, we studied the optical properties of symmetrically arranged microspheres with regards to coupled resonance modes and directional narrow beam emission by means of photonic nanojets [10]. The unique feature of nanojets is a directional beam with a beam waist smaller than the diffraction limit. We observed strongly directed far field emission in 3-sphere, 5-sphere and 7-sphere structures, which we attribute to photonic nanojets. The directional emission from the PM matches the symmetry of the structure. Independent of the photonic nanojets, we investigated if WGMs develop within the multisphere structures. We observed uncoupled modes as well as evidence of coherently coupled modes which are present in the PM.

\section{EXPERIMENTAL METHOD}

We carried out spectral measurements by exciting the dye-coated spheres and taking the photoluminescence spectrum of a 3-sphere triangular PM and the 5- and 7-sphere cyclic PM. To form the symmetric PMs, the microspheres were placed on a solid Si-wafer substrate. Due to electrostatic force between the spheres, the microspheres aglomerate into different configurations. The microspheres used in this experiments are made of melamine formaldehyde latex $(n=1.68)$ with a diameter of $5.374 \mu \mathrm{m} \pm 0.069 \mu \mathrm{m}$ according to specifications from MicroParticles $\mathrm{GmbH}$. The microspheres are coated with Rhodamine 6G fluorescence dye. The CCD images of the emission field and the spectral measurements were taken with a commercial Renishaw microphotoluminescence/micro-Raman machine.

\section{RESULTS AND DISCUSSION}

The spectra of a single $5.374 \mu \mathrm{m}$ sphere and the coupled 3-sphere triangular structure are shown in Fig. 1. The spectrum in Fig. 1a reveals the resonance peaks of the TE and TM modes of a single microsphere. Detailed analysis of mode structure in these single microspheres is presented elsewhere [9]. The fine unsplit peaks in the single sphere spectrum possess a linewidth of about $0.2 \mathrm{~nm}$. That corresponds to a Q-factor of about Q $\approx 2900$. The spectrum of the triangular structure is shown in Fig. 1b. The resonance mode distribution is rather complex, mainly due to higher order modes and the size difference of the microspheres. The spectrum shows resonances which can be assigned to modes in the single sphere spectrum. The additional resonances indicate coupled 
modes that have developed in the PM structure due to strong coherent coupling between the spherical microcavities.

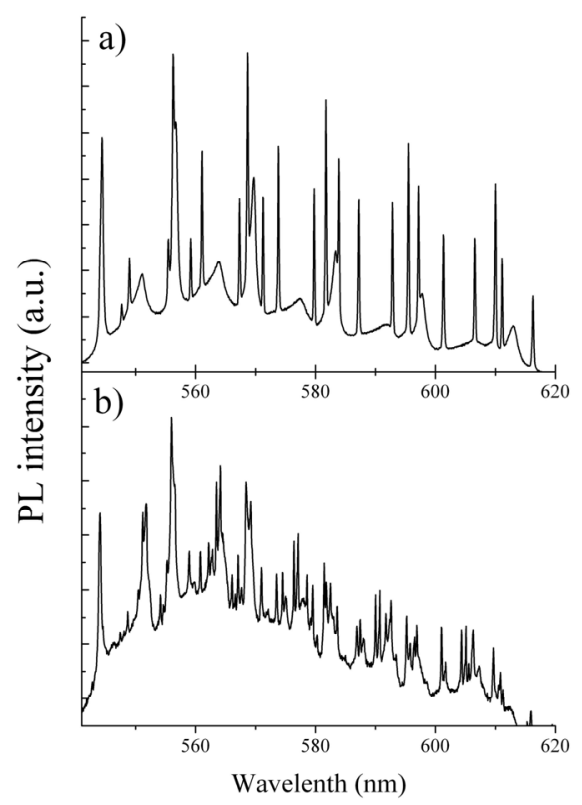

c)

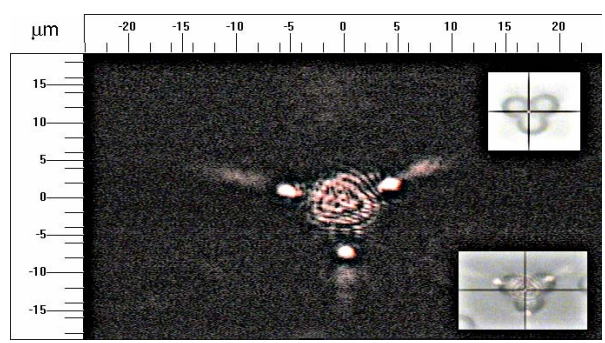

d)

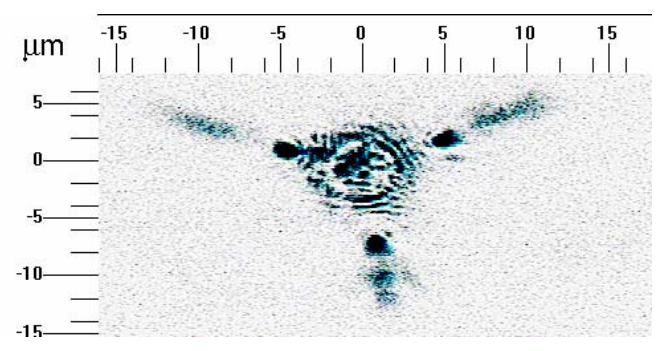

Figure 1.(a) Spectrum of a single microsphere with a nominal diameter of $5.374 \mu \mathrm{m}$. (b) Spectrum of a triangular photonic molecule consisting of 3 microspheres. (c) Photonic nanojets generated by laser illumination of a triangular photonic molecule. Inset top right: Image of the tri-sphere photonic molecule in white light. The crosshair indicates the focus position of the laser. Inset bottom right: A merged image of the triangular structure under laser illumination and under white light. (d) Image shown in inverse colours. Dark colours indicate high intensities.

The directional emission of a triangular PM is shown in Fig. 1c. The laser is focused $7 \mu \mathrm{m}$ above the Si-substrate in the z-direction centred in the crosshair as shown in the top inset of Fig. 1c. The defocused beam allows coupling of the laser light into the whole structure, as the high-NA $(\mathrm{NA}=0.9)$ objective results in strong divergence of the focused beam. The position of the focus was adjusted for optimal coupling as monitored with the CCD camera. The bottom inset in the left image of Fig. 1c shows a merged image of the triangular structure in white light and the laser illuminated image. The overlapped image shows the position where the emission developed within the triangular PM is located. As the focus position of the laser and the focus plane of the CCD camera in the micro-photoluminescence setup cannot be adjusted separately, the image of the emission is not exactly in focus. Still, the image quality is sufficient to determine details in the scattered emission. The radii of the individual spheres are about $8 \lambda$. According to calculations based on Mie-theory [10], a sub- $\lambda$ nanojet on the surface of a microsphere can be obtained from spheres with a radius of about $R \approx 5 \lambda$ and a refractive index of $n \approx 1.6$. The images in Fig. $1 \mathrm{c}, \mathrm{d}$ clearly show directional emission from all three microspheres at the outer surface of the individual spheres in the structure. Most remarkable are the emission spots with high intensity located near the surface of the spheres (see bottom inset). The inverted colour image in Fig. 1d also brings out features of the internal intensity distribution within the PM. The observed intensity pattern shows similarities to field calculations carried out in $[10,11]$. Our experiment shows that the PM consisting of three symmetrically arranged microspheres can distribute a single excitation beam into three well-defined directional beams.

The spectrum of the 5-sphere ring PM structure is shown in the bottom graph of Fig. 2b in comparison with the spectrum of the single sphere (Fig. 2a). Also in comparison with the 3-sphere triangular spectrum (Fig. 1b), we observe a rearrangement of the modes. Instead of narrow resonances, broad uneven-shaped peaks develop. There are no sharp peaks which can be assigned to modes of the single sphere spectrum.

As Fig. 2c shows, the 5-sphere ring emits 5 nanojets at each sphere surface symmetric to the shape of the PM. In this structure, the laser is focused in the crosshair $6.5 \mu \mathrm{m}$ above the Si-substrate. The reflections of the nanojets on the substrate appear at around a distance of 3 to $4 \mu \mathrm{m}$ from the microspheres. Each sphere also shows the bright intensity spot at the surface from where the photonic nanojets emerge outwards symmetric to the structure.

Fig. 3b shows the spectrum of the 7-sphere PM structure in comparison with the spectrum of the single sphere (Fig. 3a). The broad peaks are more pronounced and smoother in shape. The peaks possess a linewidth of about $2 \mathrm{~nm}$. That means that the Q-factor is about one magnitude smaller than in the single sphere modes. Due to the size disorder of all 7 spheres, the linewidth is altered by inhomogeneous broadening as a result of the 
overlapping resonances. The individual modes are also broadened as a result of the direct contact between the spheres. The more spheres are arranged in a PM, the more contact regions between the spheres occur and as a result, a lowering of the Q-factor of the optical resonances takes place. The most interesting feature of the 7 -sphere system is that the modes are evenly spaced across the spectrum, indicating clearly that coherent mode coupling within the photonic molecule is indeed occurring.

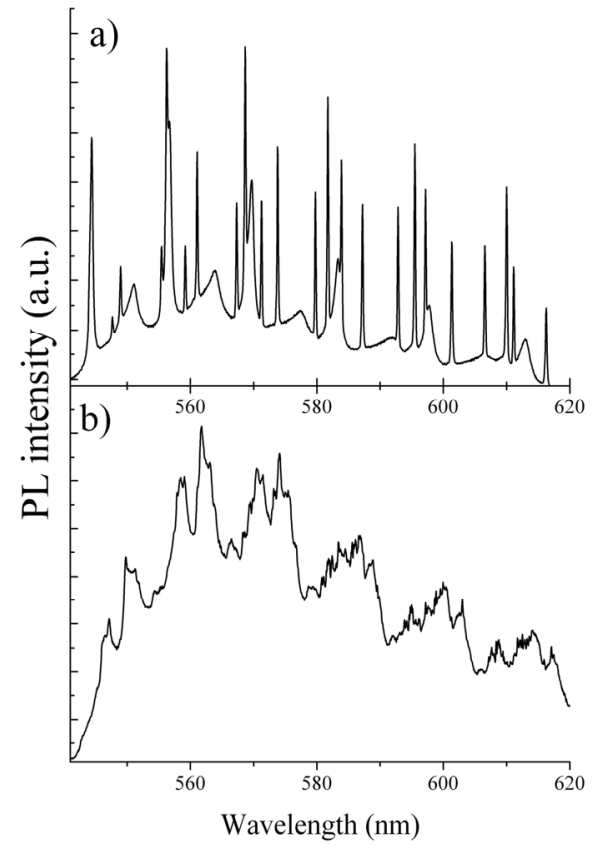

c)

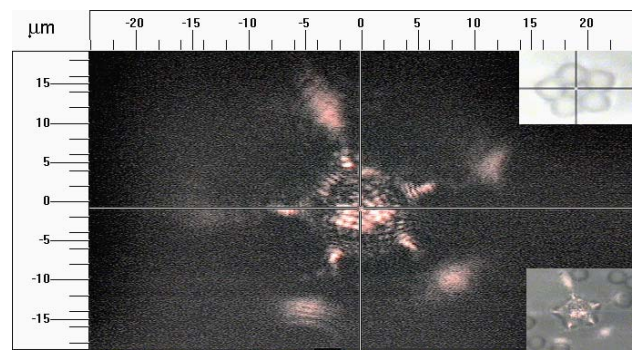

d)

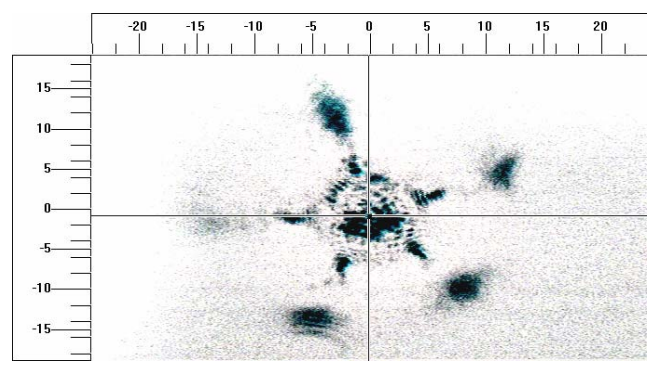

Figure 2. (a) Spectrum of a single microsphere with a nominal diameter of $5.374 \mu \mathrm{m}$. placed on a Si-wafer substrate. (b) Spectrum of a 5-sphere cyclic photonic molecule. (c) 5-sphere ring photonic molecule without a sphere in the centre. (d) Image shown in inverse colours.

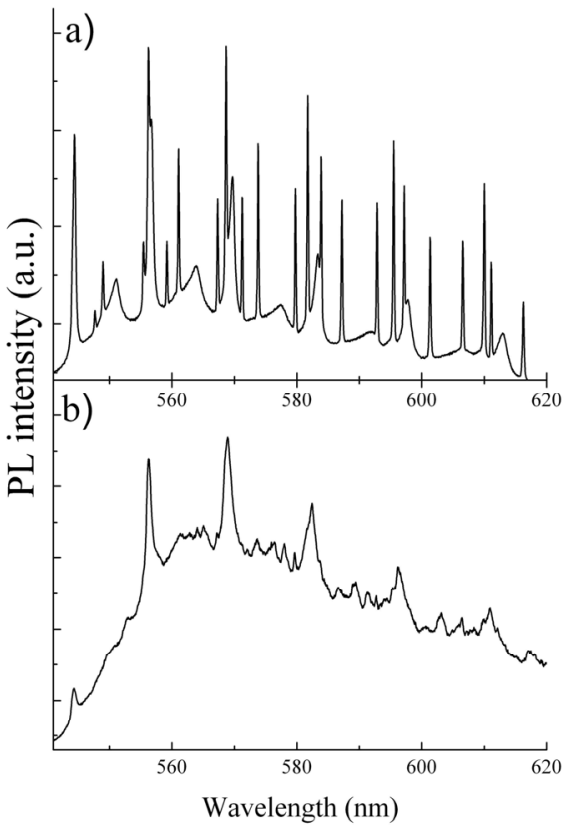

c)

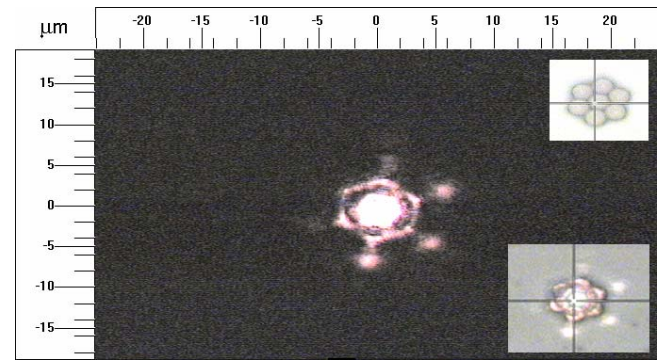

d)

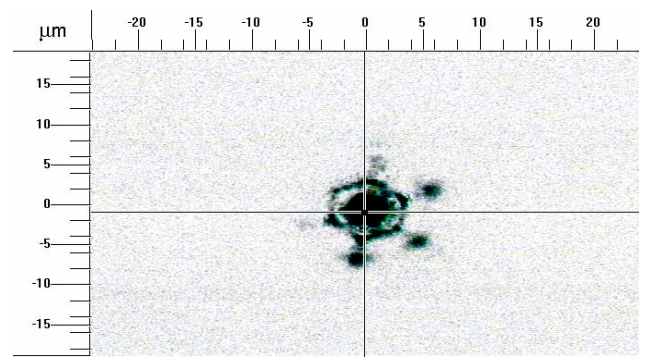

Figure 3. Spectrum of a single microsphere (a) and 7-sphere cyclic photonic molecule (b). (c) Image of 7-sphere cyclic photonic molecule in white light. (d) Image shown in inverse colours.

The illuminated structure is shown in Fig. 3c and Fig. 3d. In agreement with the photonic molecules shown above, we observed nanojet emission from each of the outside spheres. The reflection of the nanojet beams on the Si-substrate shows a round and less elongated shape compared with the 5 microsphere PM. The most outstanding feature of this structure is the field intensity within the PM. The detected light forms a hexagonal 
shape with a strong resemblance to the electron cloud distribution in a chemical benzene molecule. The structure was illuminated in the centre with a defocused beam $8 \mu \mathrm{m}$ above the substrate. Spectral analysis of the scattered light should reveal if the illuminated PM develops strong optical coupling between the spheres.

Studies of the spectral data of photonic benzene based on coupled defects in a photonic crystal were carried out by Lin [12] and Sievenpiper and co-workers [13]. The molecule orbitals are similar to the anti-bonding and bonding modes in coherently coupled microcavities. Calculations for photonic benzene [12] reveal a frequency spectrum similar to the $\pi$-molecule orbitals and the $\sigma$-molecule orbitals in the chemical molecule. The optical resonances show non-degenerate and degenerate modes in the spectrum of the coherently coupled resonators [13]. Spectral analysis of our 7-sphere structure could clarify if the hexagonal intensity distribution is related to supermodes.

\section{CONCLUSIONS}

In conclusion, the spectral analysis of the scattered light revealed WGMs in the spectrum attributed to overlapping uncoupled modes and the formation of broad peaks, indicating coherent coupling between the spheres of the PM. We have demonstrated directional emission from symmetric PMs of various shapes which we attribute to the formation of photonic nanojets. The PMs were illuminated perpendicular to the molecule plane with a defocused laser beam. Remarkable far-field emission was observed in these symmetric PM structures including a photonic benzene-like structure. The directed emission suggests the possible use of these photonic structures as a beam splitter with a number of ports corresponding to the number of spheres arranged in the photonic molecule. Photonic nanojets may allow coupling of light from the PM into other photonic micro- or nanostructure such as coupled resonant optical waveguides or nanostructures such as nanocoaxial cables [14], which are sub-wavelength optical waveguides. This would be possible not only with sub-wavelength spatial accuracy, but also with extremely high intensities in comparison with conventional sub-wavelength coupling techniques using scanning-near-field optical microscopy (SNOM)-tips.

\section{ACKNOWLEDGEMENTS}

This work has been supported by Science Foundation Ireland through the CRANN Research Centre, project PR23.

\section{REFERENCES}

[1] K. J. Vahala: Optical microcavities, Nature, vol. 424, pp. 839-846, Aug. 2003.

[2] M. Bayer, et al.: Optical modes in photonic molecules, Phys. Rev. Lett., vol. 81 pp. 2582-2585, Sept. 1998.

[3] Y. P. Rakovich, et al.: Fine structure of coupled optical modes in photonic molecules, Phys. Rev. A, vol. 70, pp. 051801-1/4, Nov. 2004.

[4] S.V. Boriskina: Theoretical prediction of a dramatic Q-factor enhancement and degeneracy removal of whispering gallery modes in symmetrical photonic molecules, Opt. Lett., vol. 31, pp. 338-340, Feb. 2006.

[5] E. I. Smotrova, et al.: Threshold reduction in a cyclic photonic molecule laser composed of identical microdisks with whispering-gallery modes, Opt. Lett., vol. 31, pp. 921-923, April 2006.

[6] S.V. Boriskina: Spectrally engineered photonic molecules as optical sensors with enhanced sensitivity: a proposal and numerical analysis, J. Opt. Soc. Am. B, vol. 23, pp. 1565-1573, Aug. 2006.

[7] S. V. Boriskina, et al.: Photonic molecules made of matched and mismatched microcavities: new functionalities of microlasers and optoelectronic components," Proc. SPIE, vol.6452, pp. 64520X-1/10, 2007.

[8] Z. Chen, et al.: Photonic nanojet enhancement of backscattering of light by nanoparticles: a potential novel visible-light ultramicroscopy technique, Opt. Exp., vol.12, pp. 1214-1220, Feb. 2004.

[9] Y.P. Rakovich, et al.: Whispering Gallery Mode Emission from a Composite System of CdTe Nanocrystals and a Spherical Microcavity, Sem. Sci. Tech., vol. 18, pp. 914-918, Aug. 2003.

[10] S. Lecler, et al.: Properties of a three-dimensional photonic jet, Opt. Lett., vol. 30, pp. 2641-2643, Oct. 2005.

[11] A. V. Itagi and W. A. Challenger: Optics of photonic nanojets, Opt. Soc. Am. A, vol. 22, pp. 2847-2858, Dec. 2005.

[12] B.S. Lin: Variational analysis for photonic molecules: Application to photonic benzene waveguides, Phys. Rev. E, vol. 68, pp. 036611-1/8, Sept. 2003.

[13] D.F. Sievenpiper, et al.: 3D Wire Mesh Photonic Crystals, Phys. Rev. Lett., vol. 76, pp. 2480-2483, Apr. 1996.

[14] T. Thio: Coaxing light into small spaces, Nat. Nanotech., vol. 2, pp. 136-138, March, 2007. 